\title{
Atomoxetine Treatment for Attention Deficit and Hyperactivity Disorder Symptoms in a Child Who Has Mucopolysaccharidosis Type IIIB Disorder
}

\author{
Cagatay Ugur ${ }^{\mathrm{a}}$, Sabide Duygu Tunas ${ }^{\mathrm{a}}$, Ozden Sukran Uneri ${ }^{\mathrm{a}}$, Zeynep Goker ${ }^{\mathrm{a}, \mathrm{c}}$, \\ Miray Cetinkaya ${ }^{b}$
}

\begin{abstract}
Mucopolysaccharidosis type III (MPS-III, Sanflippo syndrome) is one of the lysosomal storage diseases characterized by four different enzymes playing a role in catabolism of heparan sulfate substance. Type IIIB (Sanflippo B), one of them, is characterized by alpha-Nacetylglucosaminidase (NAGLU) enzyme deficiency and results in accumulation and storage of heparan sulfate in central nervous system, and then emerges itself with developmental deficiency, coarse facial features, delay in speech, mental retardation, hyperactivity, impulsivity, aggressive and disruptive behaviors, difficulty in establishing social relations, aimless hand movements, inadequate eye contact, and sleep disturbances. There are a few studies related to the treatment of neuropsychiatric symptoms of these patients. In this case, atomoxetine treatment added was shown to be effective, safe and tolerable, because risperidone treatment that the patient took was not effective in hyperactivity symptoms. A 12-year-old girl, diagnosed with MPS type IIIB (NAGLU deficiency; OMIM\# 252920) when she was 5 years old, was admitted to our clinic with symptoms of hyperactivity, short span of attention, aggressive behavior, and quasi-autistic symptoms such as aimless hand movement, with risperidone use at $1.5 \mathrm{mg}$ /day for approximately 1 year. After clinical and psychometric examinations, she was diagnosed with "mild mental retardation" and "attention deficit hyperactivity disorder". Atomoxetine was added at a dose of $0.5 \mathrm{mg} / \mathrm{kg} /$ day to her risperidone treatment and its dosage was gradually increased up until to $1.4 \mathrm{mg} / \mathrm{kg} /$ day. Over the followup period, her attention deficit and hyperactivity disorder (ADHD) symptoms were ameliorated, reported by both her teachers and her parents via rating scales of ADHD. Her hyperactivity and irritability levels were noticed as decreased and improved over evaluation process throughout clinical interviews. Through atomoxetine treatment process, there was not any adverse effect.
\end{abstract}

Manuscript accepted for publication February 22, 2016

${ }^{a}$ Ankara Pediatric Hematology Oncology Training and Research Hospital, Child and Adolescent Psychiatry Department, Turkey

bKastamonu Dr. Munif Islamoglu National Hospital, Child and Adolescent Psychiatry Outpatient Clinic, Turkey

${ }^{\mathrm{c} C}$ Corresponding Author: Zeynep Goker, Ankara Pediatric Hematology Oncology Training and Researc Hospital, Child and Adolescent Psychiatry Department, Turkey. Email: zeynepgoker@hotmail.com

doi: http://dx.doi.org/10.14740/ijcp237w
Keywords: MPS type IIIB; Hyperactivity; Quasi-autistic symptoms; Risperidone; Atomoxetine

\section{Introduction}

Mucopolysaccharidosis type III (MPS-III) disorder, also named Sanflippo syndrome, is one of the autosomal recessive neurodegenerative lysosomal storage disorders occurring in deficiency of four different enzymes which play a role in heparan sulfate catabolism [1].

In the case of alpha-N-acetylglucosaminidase (NAGLU), one of these enzymes, deficiency of heparan sulfate accumulates in systems especially in the central nervous system and the clinical features of MPS type IIIB (Sanflippo IIIB) emerge [2]. MPS-IIIB is characterized by delay in speech, mental retardation, hyperactivity, aggressive behavior, incapability in social interaction skills, aimless hand movements, inappropriate and limited eye contact, sleep disturbances, retardation in growth, and facial phenotypic features [3].

Behavioral problems are a mostly seen feature in persons with MPS-III disorder compared to the other MPS subtypes. In these children, some features of psychiatric symptoms might be seen such as severe hyperactivity, uncontrolled impulsivity, temper tantrums, quasi-autistic patterns, and severe anxious mood [4]. These symptoms have changeable natures in which some of them might be lessened and some could increase and worsen [3]. Many studies related to this disorder demonstrated that patients with MPS-III have experienced sleep disorders, hyperactivity, aggressive behavior, inappropriate mood, temper tantrum and behavioral problems similar to this and there was a correlation between cognitive retardation and behavioral problems [5].

There are lesser extent of studies in connection with the treatment of the psychiatric symptoms and disorders in patients with Sanflippo syndrome. With very limited nature, antipsychotics, especially risperidone, have been seen as effective in behavioral symptoms and hyperactivity was seen in children with MPS-III [6]. In this recent case, a patient with MPS-III took risperidone treatment because of her hyperactivity but there was no improvement. After examination of hyperactivity, short attention time, and uncontrolled impulsivity, we added atomoxetine to recent risperidone treatment. On follow-up, it 
was noticed that all symptoms of hyperactivity, inattention and impulsivity were ameliorated.

\section{Case Report}

A 12-year-old girl was admitted to our clinic with complaints of hyperactivity, short span of attention, aggressive behavior, delay of speech, and pointless hand movement.

\section{History}

She was a child of a 33-year-old mother who graduated from high school, with first delivery, after normal pregnancy span, with normal spinal-vaginal route, and weight as 3,500 $\mathrm{g}$ in a hospital. There were no highlighted issues in postnatal period like jaundice, febril convulsion, or staying in a neonatal incubator.

There was no kinship relation between mother and father. There was no specific issue in her toddler age or any specific pathological medical condition. When she was 5 years old, symptoms emerged such as regression and retardation in her speech skills that once she achieved and sphincter control that she gained before. With adding symptoms like inappropriate social interactions she displayed, distractibility, hyperactivity, and impulsivity to her recent features, clinical and genetic examinations were carried out.

Her neurological and radiological examinations were found normal, and cranial MRI and EEG were also normal. Genetic analysis showed NAGLU deficiency was seen in MPS IIIB (OMIM\# 252920) and then she was diagnosed with MPS type IIIB. Also she was diagnosed with mild mental retardation and she was steered to a special education programme when she was 5 years old.

There was also no history of using medication until she was 11, when her hyperactivity was worsened and aggressive behaviors reached intolerable levels. Risperidone treatment was begun to treat for these syptoms with a beginning dose of $0.25 \mathrm{mg} /$ day and then progressively was increased up to 1.5 $\mathrm{mg}$ /day since it was not enough for her hiyperactivity and aggressive symptoms.

The only specific issue we found in her family history was that there was a mental retardation in her first cousine (a boy), and an epilepsy in her another first cousine (a girl).

\section{Phenotypical features}

Physical examination of the case revealed coarse facial features with dysmorphic characteristics including hypertelorism and mildly high-arched palate, for which Child Neurology and Child Metabolism Disorders Departments were consulted.

\section{Developmental stages}

In questioning for her gross motor skill abilities, it was detect- ed that she could hold her head erect when she was 2 months old, sit without any supported equipment by 8 months of age, and walk when she was 14 months old. She began to talk with one or two words when she was 18 months old. She acquired her sphincter controls when she was in 4 years of age. From this year of age onwards, incapabilities in displaying social interactions, progressively loss of abilities and skills of developmental stages that once they had gained, and limited and superficial eye contact that she displayed disturbed her parents and then they sought an explanation and then they went to a hospital.

\section{Psychiatric evaluation}

The patient's first examination for evaluating her symptoms was carried out by our clinic when she was 12 years old, taking regularly $1.5 \mathrm{mg} /$ day risperidone psychotropic agent.

Psychiatric examination revealed that she had limited and superficial eye contact, and very limited non-verbal communication means such as gesture and mimics. She had inappropriate level of social interaction skills compared to her peer group (as normative). She had poorly joint attention which was inconsistent and inappropriate. She was not capable of responding to social and emotional interactions and she could not be able to establish appropriate eye contact after calling her with her name. She did not display her age-appropriate thematic plays and stereotypic movements like hand clapping. Although she had been taking risperidone medication for a long time, she displayed hyperactivity and aggressive behavior, increased impulsivity, disorganization, and poor adaptation skills which affected her functioning domains that she needed on a daily basis. We noticed quasi-autistic symptoms in this case. After the clinical and psychometric examinations, she was diagnosed with "mild mental retardation" and "attention deficit hyperactivity disorder", guided by DSM-5 criteria [7].

Atomoxetine was added at a dose of $0.5 \mathrm{mg} / \mathrm{kg} /$ day to her recent $1.5 \mathrm{mg} /$ day risperidone treatment protocol and its dosage was gradually increased up until to $1.4 \mathrm{mg} / \mathrm{kg} /$ day. She was followed up at first month, second month, and sixth month. Over follow-up period, her attention deficit and hyperactivity disorder (ADHD) sypmtoms were ameliorated, reported by both her teachers and her parents via rating scales of ADHD. Her hyperactivity and irritability levels were noticed to decrease and improve over evaluation process throughout clinical interviews. Through atomoxetine treatment process, there was not any adverse effect.

To continue the specific education programme, her medical record was updated. For improving the social interaction skills and communication abilities, she was recommended with another specific education programme designed for autistic children to support her. Over follow-up period, it was noticed that both specific education programmes for mental retardation and for quasi-autistic symptoms were benefical to her (reported by both her teachers from education centers and by her parents). Atomoxetine dose was fixed at $1.4 \mathrm{mg} / \mathrm{kg} /$ day and continued. Periodical follow-up was planned with specific intervals. 


\section{Discussion}

To discriminate between behavioral problems seen in MPS and ADHD or autism spectrum disorders is likely difficult to clinicians [5]. Since in many patients who had MPS severe behavioral problems are prominent and severe hyperactivity is a very characteristic feature seen [8]. Some authors even argued that behavioral and psychiatric symptoms seen in the case with MPS type IIIB could misguide the clinicians to misdiagnose such as autism or ADHD to the children with MPS type IIIB and then to attempt to treat these disorders mistakenly [9].

Amongst the patients with MPS type III, the severity of the disorder varies. Thus, there could be different treatment protocols for these different levels of symptoms which patients have child by child.

Besides, psychiatric symptoms and other comorbid disorders found might very well affect the quality of life of both patients and their parents and could cause the disruption in adherence to treatment of the disease. Therefore, appropriate interventions carried out in time are crucially important for functioning of both patients and their parents.

There are relatively fewer studies reported concerning the prognosis and treatment of MPS type III [10-12]. There is still not a clear and proved treatment protocol to treat the core symptoms of MPS type III disorder, though the importance of early diagnosis has been highlighted in the studies reported related to this disorder [13]. Still, recent efforts to treat MPS type III are intrathecal enzyme replacement therapy and treatment protocols targeting to decrease the substrates of this disease $[14,15]$.

Although stem cell transplantation has been proved as effective in many other studies for many disorders, this was not seen to be effective in MPS type IIIA and type IIIB [1618]. Unfortunately, stem cell inocculation has not improved the neurological symptoms, which already occurred and been displayed in the clinical features of affected children. On the other hand, there are few promising animal studies pointing out that stem cell transplantation carried out by umbilical cord origin has a valuable possible effect on the features of treating procedures of MPS type III in the near future [19].

Up until now, the treatment protocol of the cases with MPS is mostly relied on to treat symptoms which affect negatively the quality of life of the affected persons and therefore, there are but supportive treatments, which have very limited characteristics. Targeted symptoms affecting negatively the quality of life in MPS type III cases to treat are aggressive behavior, increased impulsivity, irritability, stereotypic movements, and disturbances in sleep and apetite. There are, however, a few studies regarding pharmacological treatments and their effectiveness in treating symptoms is seen in MPS cases.

A study highlighted the warning label to the attention of the parents whose child has MPS that adding antipsychotics to a treatment protocol of MPS such as thioridazine or haloperidol could temporarily cause a worsening situation in unpredictable aggressive behavior of children with MPS [8]. At the same time, recent studies pointed out that risperidone, an atypic antipsychotic agent, has been well tolerated by children with MPS and has shown that it improved the problematic issues of children such as aggressive behavior, self-mutilation and temper tantrum [20]. Although risperidone has been proved as an effective, tolerable and safe agent in children with MPS type III, there are still few studies pointing out this conclusion [21].

A study conducted with MPS-IIIA cases showed a significant improvement in aggressive behavior and an important amelioration in clinical global functioning (CGF) scores of the patients [6]. There was, however, not any study related to the methylphenidate or atomoxetine treatment in cases with MPS having hyperactivity and/or irritability/impulsivity symptoms.

Atomoxetine is a well-known molecule as effective and tolerable in treating ADHD symptoms [22]. A great number of studies showed its effectiveness on irritability, aggressive behavior, short span of attention, increased impulsivity and hyperactivity $[23,24]$. Nevertheless, there was not any recent study in connection with atomoxetine treatment in MPS cases whose hyperactivity and impulsivity symptoms were prominent.

In this case, atomoxetine treatment added to risperidone improved hyperactivity symptoms, short span of attention, impulsivity, and aggressive behavior. In this respect, there is a need to explore the effect of atomoxetine on hyperactivity and attention deficiency with controlled studies. Atomoxetine was proved as effective in this MPS-IIIB case with ADHD who had risperidone treament and had no improvement in symptoms and tolerable as well.

\section{Conflict of Interest}

Authors declare that there is not any conflict of interest.

\section{References}

1. Saini AG, Singhi P, Sahu JK, Ganesan SL, Vyas S, Rao S, Sachdeva MU. Hyperactivity, unexplained speech delay, and coarse facies - is it Sanfilippo syndrome? J Child Neurol. 2014;29(8):NP9-12.

2. Elcioglu N, Pamuk O, Sari F, Beck M, Wollnik B. Sanfilippo Sendromlu Olgular?miz. J LSD. 2010;2(1):63-65 (In Turkish).

3. Valstar MJ, Marchal JP, Grootenhuis M, Colland V, Wijburg FA. Cognitive development in patients with $\mathrm{Mu}-$ copolysaccharidosis type III (Sanfilippo syndrome). Orphanet J Rare Dis. 2011;6:43.

4. Bax MC, Colville GA. Behaviour in mucopolysaccharide disorders. Arch Dis Child. 1995;73(1):77-81.

5. Wijburg FA, Wegrzyn G, Burton BK, Tylki-Szymanska A. Mucopolysaccharidosis type III (Sanfilippo syndrome) and misdiagnosis of idiopathic developmental delay, attention deficit/hyperactivity disorder or autism spectrum disorder. Acta Paediatr. 2013;102(5):462-470.

6. Kalkan Ucar S, Ozbaran B, Demiral N, Yuncu Z, Erermis $\mathrm{S}$, Coker M. Clinical overview of children with mucopolysaccharidosis type III A and effect of Risperidone treatment on children and their mothers psychological status. Brain Dev. 2010;32(2):156-161. 
7. American Psychiatric Association. Diagnostic and Statistical Manual of Mental Disorders (DSM-5). American Psychiatric Publication, 2013.

8. Cleary MA, Wraith JE. Management of mucopolysaccharidosis type III. Arch Dis Child. 1993;69(3):403-406.

9. Brady J, Trehan A, Landis D, Toro C. Mucopolysaccharidosis type IIIB (MPS IIIB) masquerading as a behavioural disorder. BMJ Case Rep. 2013;2013.

10. Ruijter GJ, Valstar MJ, van de Kamp JM, van der Helm RM, Durand S, van Diggelen OP, Wevers RA, et al. Clinical and genetic spectrum of Sanfilippo type C (MPS IIIC) disease in The Netherlands. Mol Genet Metab. 2008;93(2):104-111.

11. Meyer A, Kossow K, Gal A, Muhlhausen C, Ullrich K, Braulke T, Muschol N. Scoring evaluation of the natural course of mucopolysaccharidosis type IIIA (Sanfilippo syndrome type A). Pediatrics. 2007;120(5):e1255-1261.

12. van de Kamp JJ, Niermeijer MF, von Figura K, Giesberts MA. Genetic heterogeneity and clinical variability in the Sanfilippo syndrome (types A, B, and C). Clin Genet. 1981;20(2):152-160.

13. Rumsey RK, Rudser K, Delaney K, Potegal M, Whitley $\mathrm{CB}$, Shapiro E. Acquired autistic behaviors in children with mucopolysaccharidosis type IIIA. J Pediatr. 2014;164(5):1147-1151 e1141.

14. de Ruijter J, Valstar MJ, Wijburg FA. Mucopolysaccharidosis type III (Sanfilippo Syndrome): emerging treatment strategies. Curr Pharm Biotechnol. 2011;12(6):923-930.

15. Malinowska M, Wilkinson FL, Bennett W, LangfordSmith KJ, O'Leary HA, Jakobkiewicz-Banecka J, Wynn $\mathrm{R}$, et al. Genistein reduces lysosomal storage in peripheral tissues of mucopolysaccharide IIIB mice. Mol Genet Metab. 2009;98(3):235-242.

16. Souillet G, Guffon N, Maire I, Pujol M, Taylor P, Sevin F, Bleyzac N, et al. Outcome of 27 patients with Hurler's syndrome transplanted from either related or unrelated haematopoietic stem cell sources. Bone Marrow Transplant. 2003;31(12):1105-1117.

17. Yamada Y, Kato K, Sukegawa K, Tomatsu S, Fukuda S, Emura S, Kojima S, et al. Treatment of MPS VII (Sly disease) by allogeneic BMT in a female with homozygous A619V mutation. Bone Marrow Transplant. 1998;21(6):629-634.

18. Herskhovitz E, Young E, Rainer J, Hall CM, Lidchi V, Chong K, Vellodi A. Bone marrow transplantation for Maroteaux-Lamy syndrome (MPS VI): long-term followup. J Inherit Metab Dis. 1999;22(1):50-62.

19. Sanberg PR, Eve DJ, Willing AE, Garbuzova-Davis S, Tan J, Sanberg CD, Allickson JG, et al. The treatment of neurodegenerative disorders using umbilical cord blood and menstrual blood-derived stem cells. Cell Transplant. 2011;20(1):85-94.

20. McCracken JT, McGough J, Shah B, Cronin P, Hong D, Aman MG, Arnold LE, et al. Risperidone in children with autism and serious behavioral problems. N Engl J Med. 2002;347(5):314-321.

21. Valstar MJ, Ruijter GJ, van Diggelen OP, Poorthuis BJ, Wijburg FA. Sanfilippo syndrome: a mini-review. J Inherit Metab Dis. 2008;31(2):240-252.

22. Polzer J, Bangs ME, Zhang S, Dellva MA, Tauscher-Wisniewski S, Acharya N, Watson SB, et al. Meta-analysis of aggression or hostility events in randomized, controlled clinical trials of atomoxetine for ADHD. Biol Psychiatry. 2007;61(5):713-719.

23. Ghanizadeh A. Atomoxetine for treating ADHD symptoms in autism: a systematic review. J Atten Disord. 2013;17(8):635-640.

24. Charnsil C. Efficacy of atomoxetine in children with severe autistic disorders and symptoms of ADHD: an openlabel study. J Atten Disord. 2011;15(8):684-689. 